\title{
Infrared Emission from Interstellar Dust in the Local Interstellar Medium
}

\author{
W.T. Reach and F. Boulanger \\ Institut d'Astrophysique Spatiale, Bâtiment 121, \\ Université Paris Sud, F-91405 Orsay cedex, France
}

\begin{abstract}
In this contribution, we discuss some topics in the study of dust in the local interstellar medium. The spectrum of local clouds has recently been measured using $I S O$ and $C O B E$, confirming in general the models of excitation and cooling of dust illuminated by the the interstellar radiation field. We discuss in some detail the spatial correlation of interstellar dust and gas and the idea that infrared emission traces the total column density of the interstellar medium, with the particular application to the formation of $\mathrm{H}_{2}$. We also show that dust is present in the nearby Loop I shell, with properties similar to average, suggesting that the walls of the Local Bubble would also have relatively 'normal' dust content.
\end{abstract}

\section{Introduction}

Dust grains play crucial roles in both the thermodynamics and chemistry of the interstellar medium, but their role in the local bubble is not well known. In general, dust is observed to be well-mixed with interstellar gas for a wide range of physical conditions; however, grains are suspected to be efficiently destroyed in fast interstellar shocks, such as are thought to have formed the 'local bubble'--by which we mean the boundaries and interior of the region of low H I column density and of significant X-ray emissivity surrounding the Solar System to $\sim 100 \mathrm{pc}$. Here we address the question of whether dust grains exist in the local bubble and what their properties might be. The Ulysses spaceprobe has already detected interstellar dust flowing through the Solar System (Grün et al. 1990, Landgraf et al. 1997), so understanding the nature of interstellar dust is no longer a 'remote' astrophysical issue but rather it makes a direct connection to the Solar System. The diffuse interior of the local bubble itself (including any 'local interstellar cloud') has too low column density for its infrared emission or starlight extinction to be directly observed, but some relatively dense clouds have been identified with distances that place them in the bubble (Kerp, Herbstmeier, \& Mebold 1993). Furthermore, the extent of the 'local bubble' is comparable to the scaleheight of neutral gas in the disk. Therefore, emission from neutral phases of the interstellar medium at relatively high galactic latitudes (say $|b|>30^{\circ}$ ) has a significant probability of being part of the local bubble. 


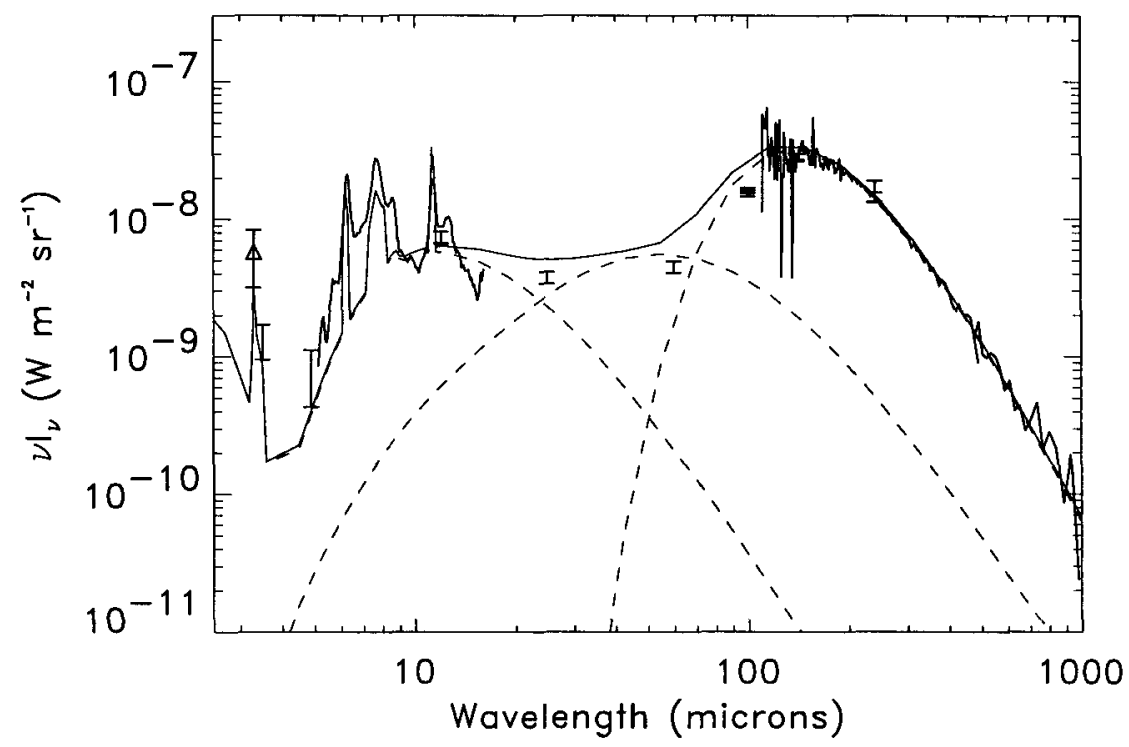

Fig. 1. The infrared emission spectrum of interstellar dust heated by the interstellar radiation field. The brightness here corresponds to an interstellar gas column density of $10^{20} \mathrm{~cm}^{-2}$. The continuous thin curve is a model consisting of three dust components, whose individual contributions - discussed in the text-are shown as thin dashed lines. The observed spectrum of interstellar dust is shown as solid curves; the far-infrared spectrum is from $C O B E$ /FIRAS, and the mid-infrared spectrum is from ISOCAM. Points with error bars are from $C O B E / D I R B E$ broad-band observations and the Arome balloon experiment.

\section{Origin of Infrared Emission}

Interstellar dust absorbs energy from starlight, and it emits this energy in the infrared. No other heating or cooling mechanisms have been found to be of comparable importance for dust grains, under typical interstellar conditions, and it is doubtful that any other mechanism (such as collisional heating by hot plasma) is important in the local bubble. The distinctive pattern of starlight extinction by interstellar dust has allowed the determination of the size distribution, and to some extent the composition, of dust particles (Mathis, Rumpl, \& Nordsieck 1977). Knowing the average value of the interstellar radiation field (Mathis, Mezger, \& Panagia 1983), the infrared emission can be calculated by a simple thermal balance for each grain size. However, it came as somewhat as a surprise, when the Infrared Astronomical Satellite (Low et al. 1984) systematically observed the infrared emission from the diffuse interstellar medium at $12,25,60$, and $100 \mu \mathrm{m}$ wavelength, that 
the straightforward estimate under-predicted the 12 and $25 \mu \mathrm{m}$ brightness by orders of magnitude. The IRAS observations led to two significant advances. First, it was realized that small grains are not in equilibrium with the relatively slowly arriving ultraviolet photons of the interstellar radiation field. A non-equilibrium model, where the dust temperature rises significantly after the absorption of an ultraviolet photon, can explain some of the bright mid-infrared emission-in particular at $25 \mu \mathrm{m}$ (Draine \& Anderson 1985). But the small size of the particles that are required to reproduce the $12 \mu \mathrm{m}$ emission makes them essentially large molecules, of which a likely candidate, polycyclic aromatic hydrocarbons (PAH), was suggested as the carrier (Leger \& Puget 1984).

The infrared emission spectrum calculated by a 'modern' model for the interaction of interstellar dust with the interstellar radiation field is shown in Figure 1. The model shown here, taken from Désert, Boulanger, \& Puget (1990), includes three components of the grain population:

- big grains in thermal equilibrium with the radiation field dominate the far-infrared emission;

- very small grains stochastically heated by ultraviolet photons dominate the emission between 30 and $80 \mu \mathrm{m}$; and

- polycyclic aromatic hydrocarbons (PAHs) produce the lines and shortestwavelength infrared continuum.

The optical properties and size distributions in this model are based on laboratory data (optical properties of silicate and graphite), astronomical data (selective extinction and broad-band infrared colors), and a string of astrophysical inferences. Some of these inferences, for example the assignment of the far-ultraviolet extinction to PAHs and the assignment of the $2200 \AA$ bump to very small grains, remain to be tested. But the separation of the dust into three components based on characteristic size of the particle, where the interaction between the particles and the radiation field changes from classical equilibrium (big grains) to stochastic heating (very small grains) to quantum mechanical (carrier of the infrared emission bands), is likely to be a characteristic of all successful models (cf. Dwek et al. 1997).

\section{Spectroscopy of Interstellar Dust}

\subsection{Far-Infrared Spectrum}

We may now compare the models that can successfully match the broadband infrared emission with detailed spectroscopic observations. In Figure 1, we show recent spectral observations from $C O B E$ and from ISO. The farinfrared spectrum shown here was obtained by a comparison of the $C O B E$ FIRAS observations with the $\mathrm{H} I$ column density over a large fraction of the sky (Boulanger et al. 1996a). The FIRAS observed the entire sky in a $7^{\circ}$ beam, 
with very stable and calibration and good sidelobe rejection-important for measuring the faintest parts of the sky. The broad-band points in Figure 1 were obtained by correlating the COBE DIRBE (Boggess et al. 1992) skymaps with the $\mathrm{H} \mathrm{I}$ map. It is immediately evident that the far-infrared spectrum is very smooth (apart from the bright spectral line of gaseous $\mathrm{C}^{+}$, which was excised from the spectrum shown here), and a single blackbody at $17.5 \mathrm{~K}$ is a very good fit to the high-latitude spectrum (Boulanger et al. 1996a, Reach et al. 1995). The observed spectrum is in quite good agreement with the models.

\subsection{Mid-Infrared Spectrum}

Another significant advance in the observational study of the local interstellar dust is due to the mid-infrared camera aboard ISO (Kessler et al. 1996). The field of view of the camera is much smaller than that of the COBE or H I observations (compare the $7^{\circ}$ FIRAS beam to the $3^{\prime}$ ISOCAM field of view), so all-sky or even wide-field observations are out of the question. But the sensitivity of the ISOCAM is enough to detect the emission from nearby reflection nebulae and molecular clouds, even with the circular variable filter, which allows spectrophotometric maps to be made. In Figure 1 is the 4.5$17 \mu \mathrm{m}$ spectrum of a cloud near $\rho$ Oph, scaled appropriately to account for the high radiation field and column density (Boulanger et al. 1996b). We see that the mid-infrared emission from the diffuse interstellar medium is dominated by spectral lines at $6.2,7.7,8.6,11.3$, and $12.7 \mu \mathrm{m}$, and the spectrum is almost exactly the same in radiation fields ranging from the average interstellar radiation field (Chamaeleon clouds) to 50 times this ( $\rho$ $\mathrm{Oph}$ ) to $10^{3}$ times this (NGC 7023). In very strong and hard radiation fields, the mid-infrared spectrum does change (Roelfsema et al. 1996), but there is a nearly 'universal' spectrum away from hot stars. The fact that the spectrum is dominated by the emission bands clearly demonstrates that the interaction of the smallest particles in the interstellar medium is quantum mechanical. The energy absorbed in these quantum mechanical modes is not trivial in comparison with that absorbed by larger grains; to produce the observed infrared emission, the PAH must absorb about $1 / 3$ as much energy from the interstellar radiation field as the grains. The abundance requirements of the infrared band carriers is also non-trivial, with current estimates indicating about $20 \%$ of the total interstellar C being locked in PAH (Dwek et al. 1997).

\section{Relationship Between Interstellar Dust and Gas}

The fact that the interstellar dust and gas are well mixed is demonstrated by the relatively tight correlation of infrared emission with $\mathrm{H} \mathrm{I} 21-\mathrm{cm}$ column density for lines of sight away from star forming regions and other molecular clouds. This correlation is not just a reflex of the fact that both the dust and gas are components of the disk-which would cause both to scale (to 
first order) as the cosecant of galactic latitude-but rather the dust and gas are correlated in detail. This was shown on large scales by dividing both the infrared emission and $\mathrm{H}$ I column density maps (at half-degree resolution) by the cosecant of latitude; these normalized maps exhibited the same correlation as the overall emission (Boulanger and Pérault 1988). Examples of the infrared-H I correlation are shown in Figure 2. A similar comparison of high-latitude microwave emission (from the COBE DMR) with infrared maps suggests that free-free emission from diffusely-distributed H II is also correlated with dust (Kogut et al. 1996)-although the separation of dust between $\mathrm{H} \mathrm{I}$ and H II phases remains to be explored. On smaller angular scales, where high-resolution $\mathrm{H}$ I observations have been performed, the correlation with infrared emission remains strong (Joncas, Boulanger, \& Dewdney 1992) and with similar slope, suggesting that the dust-to-gas ratio is relatively constant (at the factor of two level).
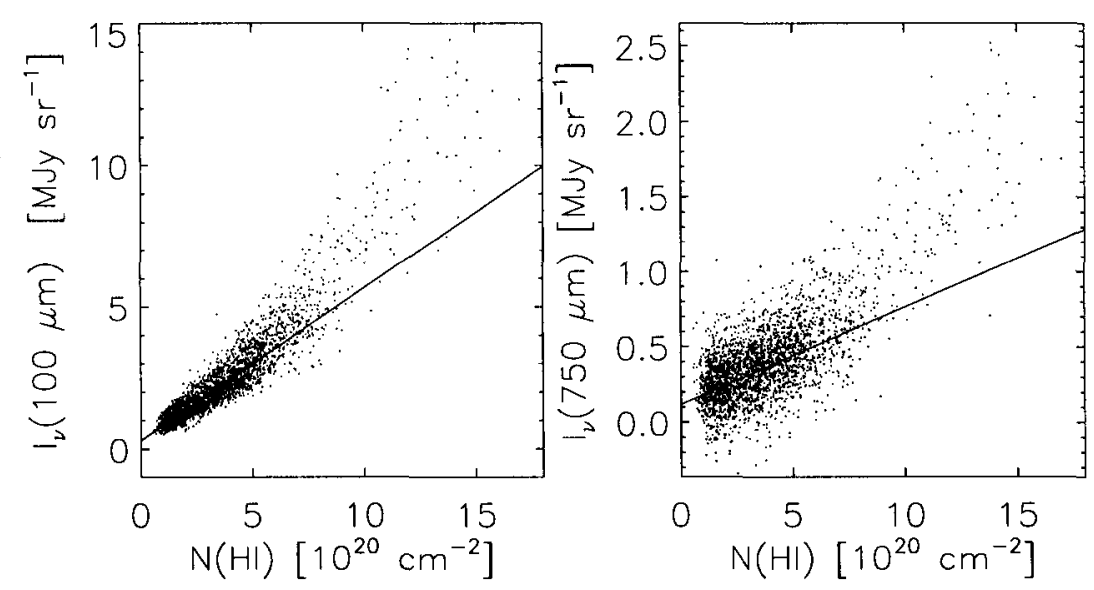

Fig. 2. Infrared surface brightness at $100 \mu \mathrm{m}$ (COBE DIRBE broad filter) and $750 \mu \mathrm{m}$ ( $C O B E$ FIRAS spectra averaged $600-900 \mu \mathrm{m}$ ) as a function of $\mathrm{H}$ I column density for the high-latitude sky (from Boulanger et al. 1996a). The straight lines are linear fits to the points with $N(\mathrm{H} \mathrm{I})<4.5 \times 10^{20} \mathrm{~cm}^{-2}$. At higher column densities the infrared emission is systematically higher than the extrapolation of the linear fits, probably due to emission by dust in molecular clouds.

We explored the constancy of the dust-to-gas ratio by comparing the $C O B E$ DIRBE $100 \mu \mathrm{m}$ map to the Leiden-Dwingeloo H I total column density in a network of patches distributed over the entire sky. It is clear that there are patches of 'excess' emission, which we will discuss below, in places where the H I column density is high. To evaluate the dust-to-gas ratio, we 
excluded pixels (which were half-degree in size) where the column density was greater than $3 \times 10^{20} \mathrm{~cm}^{-2}$. There is a range of infrared emission per unit $\mathrm{H}$ I column density, due to dust temperature variations from region to region (Désert, Bazell, \& Boulanger 1988, Reach et al. 1997). This effect is especially important at $100 \mu \mathrm{m}$ (and shorter) wavelength, where the interstellar spectrum is relatively sensitive to temperature.

\subsection{Importance of Cloud Shadows}

One perennial problem in studies of the interstellar medium is the lack of distance information. For high galactic latitudes, one generally assumes that the clouds are within a scale height, $H / \sin |b|$, of the Sun. The scale height is determined from observations of more distant gas at low latitudes and near the terminal velocity of galactic rotation (Lockman 1984). In some cases, the extinction (or $\mathrm{Na}$ or $\mathrm{Ca}$ line absorption) of light from stars at a range of distances allow one to bracket the distance to the clouds (cf. Welty, this conference). Thus some clouds are known to be relatively local, such as the 'finger' filament (Kerp, Herbstmeier, \& Mebold 1993) and MBM 12 (Hobbs et al. 1988). A significant advance in understanding the spatial relationship of the interstellar clouds and the local X-ray emitting medium was made possible by ROSAT. The sky is relatively bright in X-rays, and the absorption by even small clouds is detectable in the soft $\mathrm{X}$-rays to which ROSAT is sensitive. If there is pervasive 'background' $\mathrm{X}$-ray emission, as expected from a galactic corona (and, for very local clouds, from the local bubble emission itself), then an X-ray shadow is expected to be present either in the ROSAT all-sky survey (for places where the X-ray emission is bright) or in deeper pointed observations. It is clear that the comparison of cloud shadows, both in X-rays and in the number counts of galaxies (Finkbeiner et al., this conference), to infrared maps will shed a new light on local interstellar clouds. The optical shadows (due to dust) trace essentially all the dust, and the $\mathrm{X}$-ray shadows (due largely to He and metals) trace essentially all the gas. Thus the X-ray optical depth and the optical extinction should differ in detail from the infrared and H I maps, depending on the dust-to-gas ratio and the $\mathrm{H}$ ionization state for a particular line of sight. At this meeting it was shown in several presentations (e.g. Snowden; Mebold; Warwick; Wang) that X-ray shadows are evident essentially everywhere and from a wide range of cloud sizes. Because of the small-scale structure of the interstellar medium, and the relatively high resolution of ROSAT, the X-ray maps were often compared to infrared maps such as the IRAS $100 \mu \mathrm{m}$ maps. Our experience with the correlation between infrared emission and $\mathrm{H}$ I supports the idea that infrared emission is a tracer of the total column density of gas. However, molecular clouds have a significantly lower infrared color temperature than atomic clouds. At half-degree angular resolution, high-latitude molecular clouds have a dust temperature of $14.7 \mathrm{~K}$ compared to atomic clouds at $17.0 \mathrm{~K}$ (Reach et al. 1997); the corresponding $100 \mu \mathrm{m}$ emission per unit total gas column 
density is 3.8 times higher in atomic gas compared to molecular gas. At the higher angular resolution of IRAS and ROSAT, one will resolve even colder cloud cores; therefore, one must be cautious in the comparison of X-ray shadows (or other measures of extinction) to the $100 \mu \mathrm{m}$ emission.

\subsection{Formation of Molecular Hydrogen}

At column densities exceeding $5 \times 10^{20} \mathrm{~cm}^{-2}$, the infrared emission per unit $\mathrm{H}$ I column density is noticeably higher than at lower column densities. This is seen both in the correlation of large-scale emission (Fig. 2), within individual clouds, and for random lines of sight through clouds (Savage et al. 1977). A particularly favorable example for an individual cloud is $\mathrm{G} 236+39$, which appears on the IRAS maps as a large, centrally concentrated cloud. This cloud has been mapped in the $21-\mathrm{cm}$ line at the highest resolution possible using single-dish telescopes, the Arecibo 305-m. The infrared emission correlates perfectly with the $21-\mathrm{cm}$ column density in the peripheries of the cloud, but toward the center there is an infrared peak significantly stronger than the $\mathrm{H} I$ peak. As there is no reason to suspect that the center of this cloud is heated (in fact the $60 \mu \mathrm{m}$ emission is relatively depressed in the center of the cloud), then either there is a source of dust in the center of the cloud (which is highly unlikely because there are no $12 \mu \mathrm{m}$ sources there), or the infrared emission is due to dust associated gas in molecular form (Reach et al. 1994). Using the $C O B E$ data at 100,140 , and $240 \mu \mathrm{m}$, we confirmed that the core of this cloud is not heated, and it is only slightly colder than other dust in the region. Balancing the formation and destruction rates of $\mathrm{H}_{2}$ (van Dishoeck and Black 1986), we obtain the molecular column density of a uniform-density slab as follows:

$$
N\left(\mathrm{H}_{2}\right)=4 \times 10^{19} \chi^{-2}\left(\frac{n}{100 \mathrm{~cm}^{-3}}\right)^{2}\left(\frac{T}{80 \mathrm{~K}}\right)\left[\frac{N(\mathrm{H} \mathrm{I})}{10^{20} \mathrm{~cm}^{-2}}\right]^{2} \mathrm{~cm}^{-2}
$$

where $N$ denotes column density, $n$ volume density, $T$ the gas temperature, and $\chi$ the strength of the radiation field relative to the average ISRF at the Solar circle (Mathis, Mezger, \& Panagia 1983). Then the total column density of gas should have a quadratic dependence on the $\mathrm{H}$ I column density. (This applies only on the square-root portion of the $\mathrm{H}_{2}$ ultraviolet absorption curve of growth; at very low column densities the relation is more complicated.) The quadratic dependence was observed for G236+39, which is an isolated cloud with relatively gradual internal structure. But in general the quadratic dependence is only expected for a uniform-density region. There are two phases of atomic gas (Kulkarni \& Heiles 1988), and $\mathrm{H}_{2}$ will only form in the colder, higher-density phase; thus the total column density will be

$$
N(\mathrm{H})_{t o t}=N(\mathrm{H} \mathrm{I})+2 N\left(\mathrm{H}_{2}\right)=N(\mathrm{H} \mathrm{I})+\alpha N(\mathrm{H} \mathrm{I})_{\text {cold }}^{2} .
$$

For a large patch of sky, both cold and warm H I contribute comparable column densities, so the scatter diagram of infrared surface brightness versus 
$\mathrm{H}$ I column density has a linear lower envelope and roughly quadratic upper envelope, with individual lines of sight falling in between.

The fact that infrared emission traces the total interstellar column density, while the $21-\mathrm{cm}$ line traces the atomic gas, means that regions with 'excess' infrared emission are likely locations for molecular and ionized gas. This idea has been used to create an all-sky survey of the molecular gas (Désert, Bazell, \& Boulanger 1988), which we are currently updating using improved infrared and H I surveys. At high latitudes, the molecular gas is not diffusely distributed, because the radiation field is too strong outside of relatively dense clouds. Instead, a finite number of complexes of molecular clouds exist. Many of these have already been discovered as diffuse patches of extinction on the Palomar plates and confirmed as molecular using millimeter observations of CO lines (Magnani, Blitz, \& Mundy 1985). The infrared excess technique adds some more clouds, which may have undetectable extinction or CO lines. We have confirmed that the most significant infrared excess clouds are really regions of enhanced column density, rather than regions of warmer dust: the far-infrared colors of these regions (form the DIRBE at 100, 140, and $240 \mu \mathrm{m}$ maps) indicate that the infrared excess clouds are significantly colder than average. The combination of enhanced infrared emission, relative to $\mathrm{H} \mathrm{I}$, and relatively colder dust both indicate regions relatively shielded from the interstellar radiation field, where molecules can form. As mentioned above, the significant temperature difference between atomic and molecular gas, even at half-degree angular resolution, means that the infrared emission-especially at $100 \mu \mathrm{m}$ and shorter wavelengths - is not a linear tracer of the total column density, but this effect can be calibrated with multi-wavelength far-infrared observations (as exist on $C O B E$ and are planned for Planck surveyor).

\subsection{Shock Processing of Local Dust}

The large-scale distribution of interstellar matter consists of filaments and loops that are clearly evident in both H I $21-\mathrm{cm}$ and infrared maps. If the Solar System is within a 'local bubble,' then we can hope to understand processes that affect dust grains by studying the other, similar structures. Fast shocks can destroy large grains and shatter almost any grain, significantly modifying the size distribution (Jones, Tielens, \& Hollenbach 1996). When large grains are destroyed, the mass of very small grains must increase; comparing to the infrared emission model shown in Figure 1, we see that the $25-60 \mu \mathrm{m}$ emission should be enhanced relative to that at $100-240 \mu \mathrm{m}$. Hints of this effect have been seen in the infrared colors of clouds with a range of velocities (Heiles et al. 1988). Should the dust in the 'local bubble' have the strongly-shocked or the average size distribution?

The nearby Loop I or North Polar Spur sheds some light on this issue. The Spur is clearly evident in the nonthermal radio continuum, H I 21-cm, and infrared $60-240 \mu \mathrm{m}$ maps. We extracted profiles through the Spur at 
each wavelength and the H I map-they are very similar-and measured the following infrared colors:

$$
\begin{aligned}
& I_{\nu}(12) / I_{\nu}(100) \simeq 0.031 \pm 0.005 \\
& I_{\nu}(25) / I_{\nu}(100) \simeq 0.04 \pm 0.01 \\
& I_{\nu}(60) / I_{\nu}(100)=0.19 \pm 0.02 \\
& I_{\nu}(240) / I_{\nu}(100)=1.3 \pm 0.1 \\
& I_{\nu}(100) / N(\mathrm{H} \mathrm{I})=0.54 \pm 0.04 \mathrm{MJy} / \mathrm{sr} / 10^{20} \mathrm{~cm}^{-2}
\end{aligned}
$$

where $I_{\nu}$ is the surface brightness of Loop I minus a baseline due to more distant dust. These colors are indistinguishable from those of the average interstellar interstellar medium as shown in Figure 1. The 12 and $25 \mu \mathrm{m}$ brightness profiles are rather uncertain because the brightness of Loop I is less than $0.1 \%$ of the zodiacal light brightness at these wavelengths; however, even if the values listed here are considered as upper limits, it is still clear that the mid-infrared brightness of Loop I is not enhanced. Furthermore, the 100 $\mu \mathrm{m}$ emission per unit gas column density is indistinguishable from the average value, so the dust-to-gas mass ratio is the same. We conclude that either (1) the dust in the walls of Loop I has not been significantly processed by fast shocks, or (2) interstellar grains are significantly more resistant to sputtering and shattering than has been estimated. The same conclusions will likely apply to the walls of the 'local bubble:' we expect the grain properties to be not much different from those of the average interstellar medium.

\section{References}

Boggess, N. W., Mather, J. C., Weiss, R., Bennett, C. L., Cheng, E. S., Dwek, E., Gulkis, S., Hauser, M. G., Janssen, M. A., Kelsall, T., Meyer, S. S., Moseley, S. H., Murdock, T. L., Shafer, R. A., Silverberg, R. F., Smoot, G. F., Wilkinson, D. T., \& Wright, E. L. (1992): ApJ 397, 420

Boulanger, F., Abergel, A., Bernard, J. -P., Burton, W. B., Désert, F. -X., Hartmann, D., Lagache, G., Puget, J. -L. (1996a): A\& A 312, 256

Boulanger, F., Reach, W.T., Abergel, A., Bernard, J.P., Cesarsky, C.J., Cesarsky, D., Désert, F.X., Falgarone, E., Lequeux, J., Metcalfe, L., Pérault, M., Puget, J.L., Rouan, D., Sauvage, M., Tran, D., Vigroux, L. (1996b): A\& A 315, 352

Boulanger, F., \& Pérault, M. (1988): ApJ 330, 964

Cesarsky, D., Lequeux, J., Abergel, A., Pérault, M., Palazzi, E., Madden, S., Tran, D. (1996): A\& A 315, L305

Draine, B. T., \& Anderson, N. (1985): ApJ 292, 494

Désert, F.-X., Bazell, D., \& Boulanger (1988): ApJ 334, 815

Désert, F.-X., Boulanger, F., \& Puget, J.-L. (1990): A\& A 237, 1

Dwek, E., Arendt, R. G., Fixsen, D. J., Sodroski, T. J., Odegard, N., Weiland, J. L., Reach, W. T., Hauser, M. G., Kelsall, T., Moseley, S. H., Silverberg, R. F., Shafer R. A., Ballester, J., Bazell, D., Isaacman, R. (1997): ApJ 475, 565

Grün, E., Gustafron, B., Mann, I., Baguhl, M., Morfill, G. E., Staubach, P., Taylor, A., \& Zook, H. A. (1990): A\& A 286, 915 
Heiles, C., Reach, W. T., \& Koo, B.-C. (1988): ApJ 332, 313

Hobbs, L. M., Penprase, B. E., Welty, D. E., \& Magnani, L. (1988): ApJ 327, 356 Joncas, G., Boulanger, F., \& Dewdney, P. E. (1992): ApJ 397, 165

Jones, A. P., Tielens, A. G. G. M., \& Hollenbach, D. J. (1996): ApJ 469, 740

Kerp, J., Herbstmeier, U., Mebold, U. (1993): A\& A 268, L21

Kessler, M. F., Steinz, J. A., Anderegg, M. E., Clavel, J., Drechsel, G., Estaria, P., Faelker, J., Riedinger, J. R., Robson, A., Taylor, B. G., Ximenez de Ferran, S. (1996): A\& A 315, L27

Kogut, A., Banday, A. J., Reach, W. T., Hinshaw, G., \& Bennett, C. L. (1996): ApJ 460, 1

Kulkarni, S., \& Heiles, C. (1988): in Galactic and Extragalactic Radio Astronomy, (Springer), p. 95

Landgraf, M. et al. (1997): this volume

Leger, A., \& Puget, J. L. (1984): A\& A 137, L5

Lockman, F. J. (1984): ApJ 283, 90

Low, F. J., Young, E., Beintema, D. A., Gautier, T. N., Beichman, C. A., Aumann, H. H., Gillett, F. C., Neugebauer, G., Boggess, N., Emerson, J. P. (1984): ApJL 279, L19

Magnani, L., Blitz, L., \& Mundy, L. (1985): ApJ 295, 402

Mathis, J. S., Rumpl, W., \& Nordsieck, K. H. (1977): ApJ 217, 425

Mathis, J. S., Rumpl, W., \& Nordsieck, K. H. (1977): A\& A 128, 212

Reach, W. T., Dwek, E., Fixsen, D. J., Hewagama, T., Mather, J. C., Shafer, R. A., Banday, A. J., Bennett, C. L., Cheng, E. S., Eplee, R. E., Jr., Leisawitz, D., Lubin, P. M., Read, S. M., Rosen, L. P., Shuman, F. G. D., Smoot, G. F., Sodroski, T. J., Wright, E. L. (1995): ApJ 451, 188

Reach, W. T., Koo, B.-C., \& Heiles, C. (1994): ApJ 429, 672

Reach, W. T., Wall, W. F., Odegard, N., Weiland, J., Arendt, R. G., \& Hauser, M. G. (1997): in preparation

Roelfsema, P. R., Cox, P., Tielens, A. G. G. M., Allamandola, L. J., Baluteau, J. -P., Barlow, M. J., Beintema, D., Boxhoorn, D. R., Casinelli, J. P., Caux, E., Churchwell, E., Clegg, P. E., de Graauw, T., Heras, A. M., Huygen, R., van der Hucht, K. A., Hudgins, D. M., Kessler, M. F., Lim, T., Sandford, S. A. (1996): A\& A 315, L289

Savage, B. D., Bohlin, R. C., Drake, J. F., \& Budich, W. (1977): ApJ 216, 291 van Dishoeck, E. F., \& Black, J. H. (1986): ApJS 62, 109 